\title{
Oral Health \& Dentistry in Bhutan: call for actions to address a public health urgency
}

Karma Tenzin ${ }^{1}$, Tshewang Gyeltshen ${ }^{2}$, Gyan P Bajgai ${ }^{3}$, Sonam Nyedup ${ }^{4}$, Choki $^{5}$, Jyotsna Rimal ${ }^{6}$, OP Kharbanda ${ }^{7}$, Dechen Wangmo ${ }^{8}$, Gampo Dorji

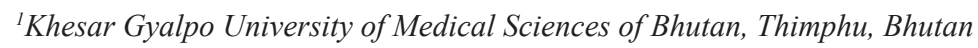

${ }^{2}$ Damphu Hospital, Damphu, Bhutan

3-5 Jigme Dorji Wangchuck National Referral hospital, Thimphu, Bhutan

${ }^{6} B P$ Koirala institute of health Sciences, Dharan, Nepal

${ }^{7}$ Indian Medical Council for research, India

${ }^{8}$ Minister for Health, Thimphu, Bhutan

${ }^{9}$ World health Organization, Delhi, India

\begin{abstract}
Introduction: Bhutan's healthcare approach in achieving the sustainable development goal 3 (SDG 3) has been largely through primary healthcare as enshrined in the universal health coverage (UHC). Bhutan has forged a unique primary healthcare model in which oral health is integrated in its primary care initiatives. The Oral Health program under the Department of public health was established in 1999 with clear line objectives. The program has achieved commendable successes over the years. However, with changing needs, enhancing and mainstreaming of oral health and dentistry in the country must be accorded a top priority. This is particularly important as the Oral Health Policy and service standards were drafted fourteen years ago in 2007. The 2021 World Health Orgnization (WHO) agreement "to provide basic oral health services to all", to which Bhutan is signatory, needs to be strictly implemented. Therefore, oral health dialogue was conducted to deliberate on the oral health systems in the country with experts from the field who were from within and outside Bhutan. This oral health policy dialogue generated important themes such as strategic and competent workforce, capacity development, need to transform oral health services in more people centric ways and mainstreaming oral health in other health policies.
\end{abstract}

Keywords: Health policy; Oral health outcome; Universal health coverage.

\section{INTRODUCTION}

Bhutan is at an epidemiological transition. The Annual Health Bulletin 2019 reported that dental caries along with diseases of teeth and gum constitute conditions with highest morbidities and continue to show an increasing trend ${ }^{1}$.

More than half of the Bhutanese population do not have access to oral health care and as result, have untreated oral and dental morbidity. It is known that a disability caused by moderate heart failure is more than a disability caused by the tooth $\operatorname{loss}^{2}$. Oral Health is a key indicator for overall health and wellbeing of a population ${ }^{3}$. A community with minimal burden of oro-dental diseases enjoy a lasting high quality of life. Therefore, to ensure that the nation's citizens enjoy a good standard of life, emphasis must be made on the provision of the best of oral healthcare services, oral health promotion, service reorientation, capacity development and policy and planning.

\section{Corresponding author:}

Karma Tenzin

karmatenzin9@gmail.com
In this regard, the Faculty of Postgraduate Medicine, Khesar Gyalpo University of Medical Sciences of Bhutan (KGUMSB) hosted Oral Health policy dialogue on $20^{\text {th }}$ March, 2021. The five imminent speakers and more than 70 participants attended the online session through Zoom conferencing platform.

\section{THEMES}

Theme 1: Oral Health Outcome and Access to Oral Health Care -impact on the poorer and lower strata section of population.

"A 30-year-old mother of two from a remote village in Tsirang has lost all her teeth to a gum disease (Periodontitis). Losing teeth has affected her health, she is frail and anemic. She does not want to get her teeth replaced from the district hospital because it requires 4 visits involving out of pocket expenditure. This is otherwise very much a preventable condition"

Access to Oral Health services has to be ensured to the whole population through Universal Health Coverage ${ }^{4}$. Implementation of effective and accessible primary oral health care services would advance health equity and strengthen integration of oral health services and in turn, help achieve the goal of universal oral health coverage. 


\section{Rank Oral disorders}

\section{Rank \\ Oral Disorders}

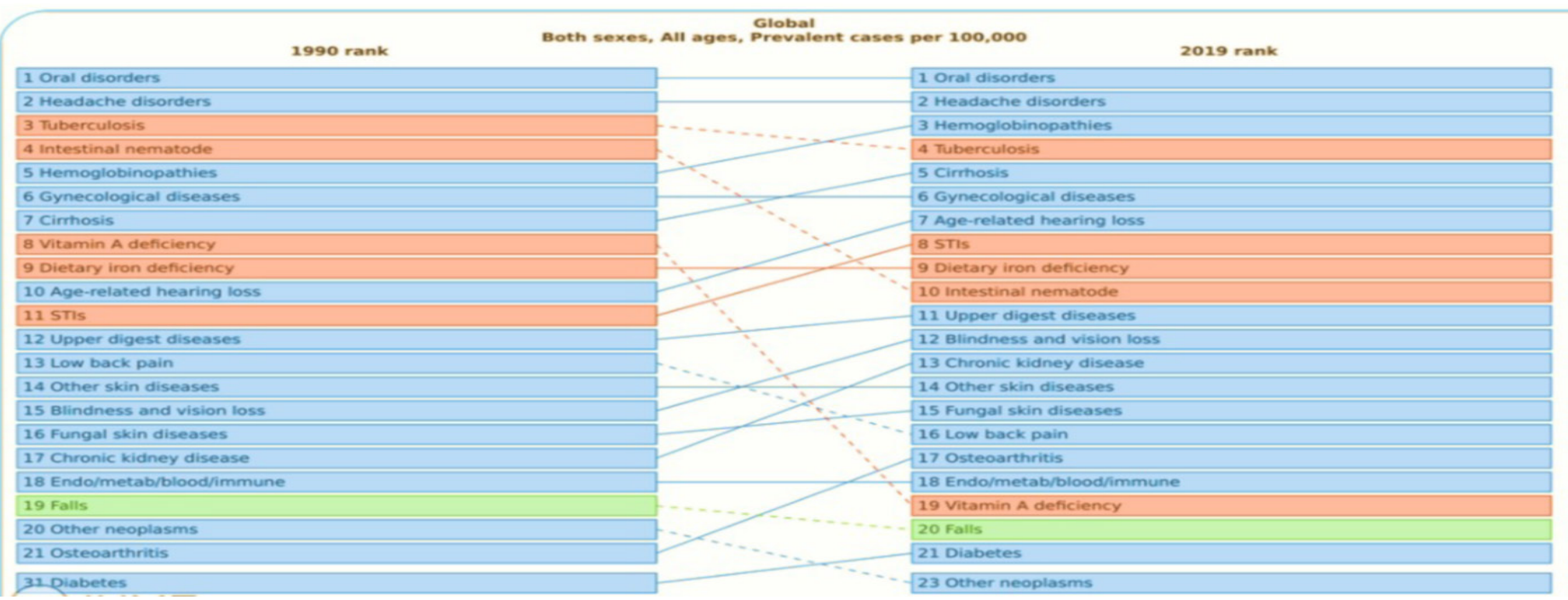

Figure 1. Comparison of oral disorders in the year 1999 to 2019. (Dr. OP Kharbanda, 2021)

The growing concern of non-communicable diseases (NCD) has increased global and national focus towards an NCD integrated policy response. Oral health could easily be part of the NCD agenda because oral health shares common biological, behavioral and psychosocial risk factors with the common NCDs. Thus, Oral Health services should be integrated into mainstream health initiatives designed to curb non-communicable diseases at the national level ${ }^{5}$.
Theme 2: Role of Oral Health services in achieving Universal Healthcare Coverage in Bhutan

The Bhutanese Healthcare system has a unique system of provision of oral and dental care services to the public ${ }^{6}$. Unlike in other countries, basic dental related services are provided free of charge across the country through the dental units in the hospitals. This uniqueness of free service delivery is one of the

\section{Trend Analysis}

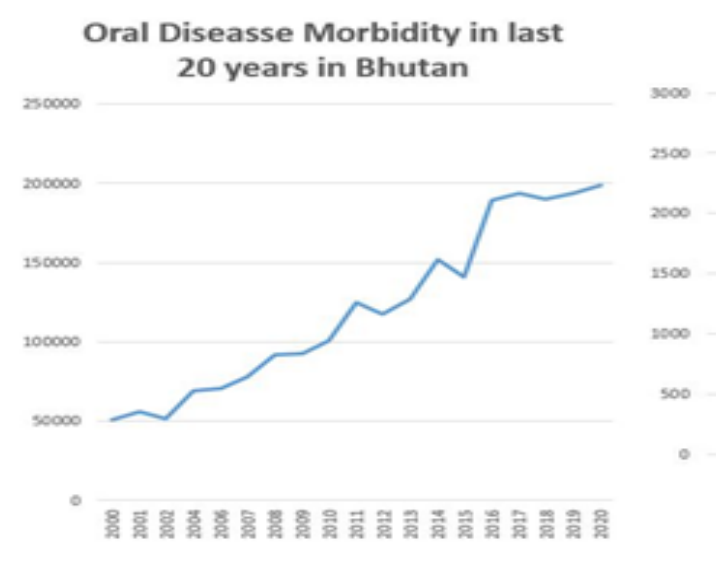

Comparative Trend to other Noncommunicable Diseases (Incidence per 10,000 population)

Figure 1. Oral Diseases Trend in Bhutan (Courtesy, Annual Health Bulletin, 2020) 
means of achieving Universal Healthcare Coverage. However, oral health and dental services are often overlooked in the context of health policy ${ }^{4}$.

In Bhutan, all the Primary Health Centers are manned by community nurses and Health Assistants. This essential group of professionals must be taken into confidence to improve implementation of preventive and advocacy measures directed towards oral health ${ }^{7}$. This could be done through the provision of basic oral examination trainings to enhance detection of suspected precancerous oral lesions, dental decays, gingivitis/ periodontitis and other similar lesions. Such trainings can also improve their competency in decision making with regards to referral to higher centers. The trainings could be conducted as on site or online institute blended learning in collaboration with the Khesar Gyalpo University of Medical Sciences of Bhutan.
Theme 3: Rethinking Oral Health workforce to address specialized care needs.

"Oral Health services needs to be commissioned based on health needs and upgrade current workforce's competency appropriately. One such approach could be to integrate basic oral health module in primary health curriculum for adoption of prevention and oral health promotion activities among the primary healthcare workers."

The country has 68 dental surgeons, 76 dental hygienists and 44 dental technicians ${ }^{9-11}$. Bhutan's overall oral health workforce distribution is 1 health worker per 11500 population, lower than WHO's recommendation of 1:7500 ${ }^{11}$. Therefore, Bhutan needs to utilize and invest in building the pool of oral health experts in a strategic manner such as establishment of dental school in the country and also designing of appropriate short courses that is aligned to the service needs of the country.

NATIONAL PROFESSIONAL DENTAL

\section{HEALTH SERVICES STANDARD}

\section{Department of Medical Services Ministry of Health \\ Thimphu \\ 2007}

\section{Acknowledgement}

The DMS and Ministry of Health would like to acknowledge the dedication and contribution of the following Health official for drafting this National Dental Health Facilities and Service Standards.

1. Dr. Samdrup R.Wangehuk Orthodentist

2. Dr. Kunzang Jigmi, Registrar, BMHC

3. Mr. Rup Narayan Chapagai, CPO, DMS

4. Mr. Senam Derji, Prog, Directer, QASD

5. Mr. Nawang Dorji, Dy. Resigtrar, BMHC

6. Mr. Tashi Penjer, Hro, RCSC

7. Mr. Tshulthrim Zangpo, HRO, MeH

8. Mr. Kinzang Thinlay, Sr. Dental Hygientist

9. Mr. Derji Wangehuk, Sr.Dental Technician

10. Mr. Deshing Lepeha, Sr. Dental Technieian

11. Ms. Phub Wangme, Dental Hygienist

Figure 3. National Dental services standard, 2007 (OP Kharbanda, 2021)

Theme 4: Strengthening the National Oral Health Policy in Bhutan

"I think you should have oral health surveillance in Ministry of Health. We need to create Bhutan Oral Health Commission (BOHC) and update the policy called Bhutan Oral Health Policy (BOHP). Your key elements have to be prevention and cost-effective treatment. You have to think about manpower, education and research."

The Oral Health program under the Department of public health was established in 1999 with clear objectives. Yet after nearly 20 years, there is a lot to achieve through focusing on appropriate technical capacity within the program at the Ministry ${ }^{4,10,12}$. This is particularly important as we envision to mainstream the Oral Health services into other non-communicable disease policy. The $2021 \mathrm{WHO}$ agreement "to provide basic oral health services to all", to which Bhutan is signatory, needs strict implementation at the national level.
Theme 5: Role of Private practice in Oral \& Dental Services Currently, Oral Health services including tooth extractions, dental fillings and consultations are provided free of cost at tertiary and secondary care levels ${ }^{4,7,9}$. Much of the specialized care services such as prosthodontic, orthodontic and Oral Medicine specialist services are also provided for free at the Jigme Dorji Wangchuck National Referral Hospital ${ }^{7,9}$. The demand for these services are very high and there are inadequate human resources to match the demand. This has resulted in a significant backlog with waiting times exceeding 12 months. This had led to many Bhutanese seeking these services in bordering Indian towns and other countries ${ }^{9}$.

The Ministry of Health should develop a policy on privatizing oral health services through regulated policies. Essential oral health services must be retained at the government hospitals. This ensures minimal out of pocket expenditure among 


\title{
.
}

\section{Oral health}

\section{Draft resolution proposed by Bangladesh, Bhutan, Botswana, Eswatini, Indonesia, Israel, Japan, Jamaica, Kenya, Peru, Qatar, Sri Lanka, Thailand and Member States of the European Union}

\author{
The Executive Board, \\ Having considered the report on oral health, ${ }^{1}$ ensuring basic oral health for all, \\ resolution: \\ RECOMMENDS to the Seventy-fourth World Health Assembly the adoption of the following
}

Figure 4. WHO resolution on basic oral health services to all (OP Kharbanda, 2021)

the poorer section of population. Provision for private clinics in cities such as Thimphu, Phuntsholing and other commercial towns must be considered. This strategic move will not only help in retaining the oral health experts within the country but also reduce the waiting time at Government hospitals. Lack of privatization suppresses the opportunity of a certain segment of the population who can and are willing to pay for the medical expenses.

Theme 6: Strategies to strengthen and build capacity of oral health professionals

The patient load and the demand for specialized dental care needs is on rise in the country. With the existing number of oral health professionals, it is a challenge to meet the demand. Therefore, a Faculty of Oral Health and Dentistry should be instituted in the Khesar Gyalpo University of Medical Sciences of Bhutan ${ }^{13}$. This will be strategic as oral health professionals will be trained within the country contextualizing local innovations and drive for academia.

\section{CONCLUDING KEY MESSAGES}

Oral Health has achieved good targets over last four decades, yet there is need to revise and refocus areas of priority in the oral health policy developments at the national level ${ }^{14}$. This is of paramount importance due to the ever evolving health needs of the country. Thus, following recommendations are submitted: 1. Improve the Oral Health Outcome and Access

a. Revisit the current oral health service standards.

b. Strategic Human Resource mappings to meet the evolving health needs

c. The National Hospital for Oral Health and Dentistry is mandated based on epidemiological, social and economic rights argument. d. Privatization of some of identified services in Dental Services through public facility encouraging user fee charges or through set up of private facilities given the current trends of the oral disease epidemiology.

2. Team based approach to Oral Healthcare services in Bhutan: The oral health programme, Academia and oral professionals must collaborate to build the capacity through adoption of various strategies such as competency based short term trainings, Specialist training with Faculty for Dentistry and Oral Health.

\section{REFERENCES}

1. Annual Health Bulletin-2020 Bhutan. Published online 2020. [Full Text]

2. Mathur MR, Williams DM, Reddy KS, Watt RG. Universal health coverage: a unique policy opportunity for oral health. J Dent Res. 2015;94(3):3S-5S. [Full Text | DOI]

3. Yap A. Oral health equals total health: a brief review. J Dent Indones. 2017;24(2):59-62. [Full Text | DOI]

4. Gyeltshen T. Oral health \& dentistry in Bhutan: call for actions to address a public health urgency. Policy Dialogue. 20th March 2021. [Full Text]

5. Fisher J, Selikowitz HS, Mathur M, Varenne B. Strengthening oral health for universal health coverage. Lancet. 2018;392(10151):899-901. [Full Text | DOI]

6. World Health Organization. The World Health Report 2008 - Primary Health Care (Now More Than Ever).; 2008. [Full Text]

7. Nyedup S. Oral health \& dentistry in Bhutan: call for actions to address a public health urgency.Policy Dialogue. 20th March 2021. [Full Text]

8. Mathur MR. Revitalizing Alma-Ata: strengthening primary oral health care for achieving universal health coverage. Indian J Dent Res. 2017;29(2):212-216. [Full Text | DOI] 
9. Baggai GP. Oral health \& dentistry in Bhutan: call for actions to address a public health urgency.Policy Dialogue. 20th March 2021. [Full Text]

10. Policy and Planning Division (Ministry of Health, Bhutan). Annual Health Bulletin 1999. Bhutan: Ministry of Health, Royal Goverment of Bhutan; 1999. [Full Text]

11. World Health Organization. The Kingdom of Bhutan Health System Review. Vol 7.; 2017. [Full Text]
12. Kharbanda OP. Oral health \& dentistry in Bhutan: call for actions to address a public health urgency.Policy Dialogue. 20th March 2021. [Full Text]

13. Rimal J. Oral Health \& Dentistry in Bhutan: call for actions to address a public health urgency.Policy Dialogue. 20th March 2021. [Full Text]

14. Asian Development Bank. Bhutan: Health Care Reform Program. Vol 1762.; 2006. [Full Text] 